Original Article $\quad$ www.pjkd.com.pk

\title{
Etiology and Outcomes of Acute Kidney Injury in Patients Admitted to a Single Tertiary Care Hospital: Balochistan Institute of Nephrology-Urology Quetta.
}

\author{
Abdul Kareem Zarkoon, Habib Ullah Rind, Moin Khan, Aijaz Ahmed, Nasir Jakrani, \\ Muhammad Hussain, Hamid Ali, Mujeeb ul Haq, Abdul Ghaffar Mandokhail, Atta Ullah, \\ Faiza Khalil, Abdul Hameed, Ghulam Muhammad.
}

Department of Nephrology, Balochistan Institute of Nephrology-Urology, Quetta, Pakistan.

\begin{abstract}
.
Introduction:

Acute kidney injury (AKI) is a common clinical syndrome with broad spectrum of etiologies and an important cause of morbidity and mortality requiring hospitalization. Depending on the cause and nature of AKI it may complicate to be life threatening or even proceed to Chronic Kidney disease (CKD) compromising the quality of life.

Methods: The current retrospective study determines the causes and outcomes of AKI in patients of different age groups, who required hospitalization at our tertiary care hospital from March 2018 to March 2020.

Possible etiologic conditions for AKI were recorded during the study period and AKI was classified according to the causes, age and outcome.

Results: records of total of 267 patients with diagnosis of AKI were obtained who were admitted during the study period. Obstetric related diagnosis was the commonest reason for AKI $(n=5018.7 \%)$, another $42(15.7 \%)$ had obstructive nephropathy, prerenal AKI in 35 patients $(13.1 \%)$ and other causes such as glomerulonephritis, sepsis, pigment nephropathy and drug related interstitial nephritis among others.

Majority of the patients needed dialytic support, $n=190$ (71.2\%) and majority of patients $n=181$ patients (67.7\%) recovered completely, and only 11 patients (4.2\%) expired.

Conclusion: Our study reveals that majority of patients with AKI presenting to BINUQ had reversible causes of AKI with complete recovery in significant number of patients. Community wise programs to early detect AKI with prompt treatment will decrease the likelihood of such patients adding to the CKD population.
\end{abstract}

Key Words: Acute kidney injury, chronic kidney disease, pigment nephropathy, post partum hemorrhage, antepartum hemorrhage, obstructive nephropathy, obstructive nephropathy, kidney stone, interstitial nephritis, hemodialysis, mortality, morbidity.

\section{Corresponding Author}

Dr. Habib Ullah Rind

Department of Nephrology

Balochistan Institute of Nephro-Urology,

Quetta, Pakistan

Email: habeebaloch@gmail.com

Received: June 27, 2020. Accepted September 25, 2020.

PJKD 2020;4(4):319-23

\section{Introduction.}

Acute kidney injury (AKI) is a clinical syndrome with rapid decline or loss of kidney function due to multiple etiological causes and an important cause of morbidity and mortality as outpatient and also in admitted patients especially admitted in the Intensive care units. The global incidence of AKI was estimated at $22 \%$ in a recent meta-analysis and 55\% among patients admitted to the ICU. ${ }^{1,2}$ The global burden of AKI is estimated 13.3 million cases per year with $85 \%$ from low middle-income countries.

The course of AKI is dependent upon many factors and one of them is early detection and intervention since it may end up in significant mortality and morbidity as CKD with or without dialysis dependency. ${ }^{4}$

The incidence and prevalence of AKI is largely dependent upon the local factors and diseases afflicting the community and are much different in underdeveloped than the developed countries. ${ }^{3.5}$ Much of it is dependent upon the available healthcare facilities, dietary habits, climate conditions and the general level of awareness among the population and health care providers. 
AKI Etiology \& Outcomes

Community acquired AKI (C-AKI) is more prevalent in developing world than hospital acquired AKI (H-AKI). Among developing countries, the leading cause is the Pregnancy related AKI (P-AKI) whereas the H-AKI is more common among the developed world.

As far as we know, no studies exist addressing AKI from Balochistan, Pakistan and the objective of this study was to determine the causes and outcomes of AKI presenting to Balochistan Institute of Nephro-Urology (BINUQ). BINUQ is the only referral center for nephrology urology related diseases catering to a large geographic area and gets referral from a wide area around Quetta and almost all parts of Balochistan province and around.

\section{Patients and Methods}

This is a retrospective study conducted at BINUQ, a tertiary care hospital, including all those cases who presented to the hospital, referred from other hospitals or admitted through OPD or emergency with AKI diagnosis. BINUQ is the only major referral tertiary care hospital in the region (Balochistan and Eastern Afghanistan) for patients with renal failure and other problems relating to nephrology and urology.

Patients were identified with the diagnosis of AKI mentioned in the medical records from March 2018 to March 2020. Records were reviewed for evaluating the cause of AKI diagnosis through history, physical examination routine hematological and biochemical investigation analysis and radiological scans including ultrasound, $\mathrm{x}$-rays, CT scans and even MRI. The serum creatinine at the time of admission and discharge were noted. Patients final discharge was evaluated for AKI recovery status (no recovery, partial recovery, completer recovery). Patients status as alive, dead or renal death requiring maintenance hemodialysis was noted.

\section{Inclusion Criteria:}

Only those patients who had AKI with their age above five years of age were included. Younger patients $(<18$ years of age) were also included since BINUQ is the only institute for kidney disease covering a wide geographical area, however they comprised only $n=13$ patients.

Patients with known mild to moderate CKD stage 01 and stage 02 with sudden rise in serum creatinine by 1.5 times in last 07 days before admission to the hospital, were included in this study.

\section{Exclusion Criteria}

Patients who initially had history of CKD stage $\geq 4$ before admission were excluded from the study for difficulty in ruling out underlying CKD progression.

All patients were categorized broadly into post-operative causes after a major surgical intervention and medical causes. Post-operative causes were defined as AKI within 7 days after the surgical procedure. Medical causes were further subcategorized into P-AKI, AKI secondary to obstructive nephropathy, stones disease, glomerulonephritis, vasculitis, pre-renal (hemorrhage, gastrointestinal loss of fluid or blood), sepsis or septic shock, pigment or toxin induce nephropathy and AKI on CKD due to any of the above causes.

\section{Statistical analysis:}

All data was entered in SPSS version 23 (IBM). Mean and standard deviation was calculated, frequency and percentage were calculated for each presentation.

\section{Results:}

A total of 324 cases were reviewed and 267 cases of AKI were included as per inclusion and exclusion criteria in this retrospective study. Age distribution revealed that only $n=13(4.8 \%)$ were $<18$ years of age, whereas majority $n=173(65 \%)$ were in the younger age group of 18- 55 years, Table 1. Frequency of AKI among all categories is described in Table 2, revealing P-AKI as the main presentation $n=50$ $(18.7 \%)$.

42 patients (15.7\%) had AKI secondary to obstructive uropathy (secondary to stones disease, BPH, any Abdominal malignancies/lymphadenopathy causing compression effect) Table 2.

Table 1: Age distribution of 267 patients with diagnosis of Acute Kidney Injury.

\begin{tabular}{|c|c|}
\hline Age Group & Frequency (\%) \\
\hline $1-18$ & $13(4.9)$ \\
\hline $18-25$ & $40(15)$ \\
\hline $26-45$ & $86(32.2)$ \\
\hline $46-55$ & 47 (17.6) \\
\hline $56-70$ & 37 (13.9) \\
\hline$>70$ & 44 (16.5) \\
\hline Total & $267(100)$ \\
\hline
\end{tabular}


AKI Etiology \& Outcomes

Table 2: Etiology of Acute Kidney Injury among 267 patients admitted to Balochistan Institute of Neprology-Urology Quetta (BINUQ).

\begin{tabular}{|l|l|}
\hline Causes & Number of Cases (\%) \\
\hline Post-Operative AKI & $31(11.6)$ \\
\hline Obs. Related AKI & $50(18.7)$ \\
\hline Obstructive Uropathy caused AKI & $42(15.7)$ \\
\hline Aki on CKD & $40(15)$ \\
\hline Pre-Renal cause of AKI & $34(12.7)$ \\
\hline Glomerulonephritis/Vasculitis & $29(10.9)$ \\
\hline Sepsis/Urosepsis & $21(7.9)$ \\
\hline Pigments/toxins/Rhabdomyolysis & $16(6)$ \\
\hline others & $4(1.5)$ \\
\hline
\end{tabular}

We further grouped the patients according to those having possible preventable AKI cause, including AKI on CKD, pre-renal, sepsis and obstruction putting together totaled $\mathrm{n}=137(51 \%)$. If we include the P-AKI also as a preventable cause, then $\mathrm{n}=148(70 \%)$ makes the major part of preventable causes of AKI, Figure 1.

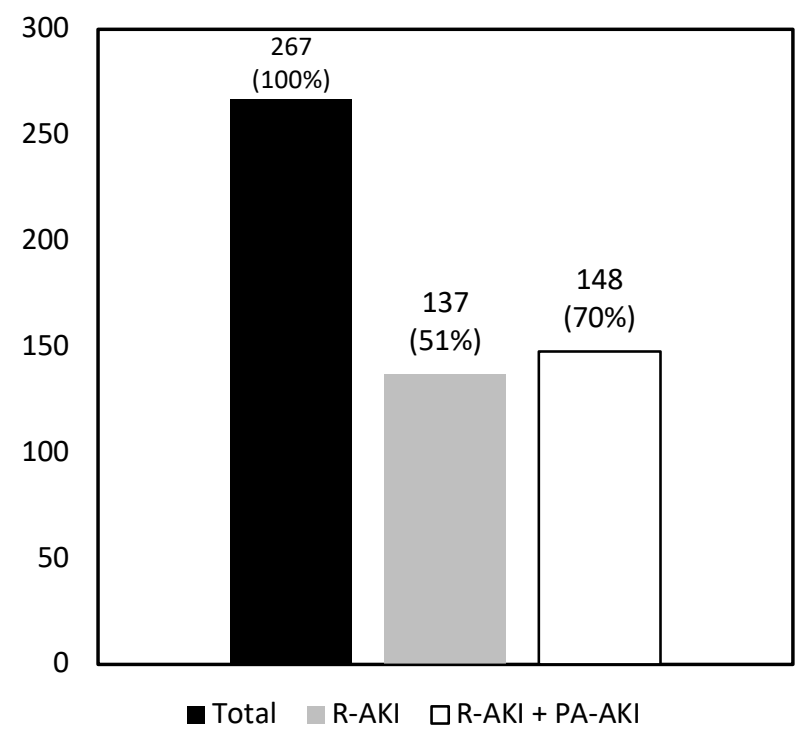

Figure 1: Bar graph showing reversible causes of AKI (R-AKI) among 267 patients admitted with AKI. The white bar shows R-AKI and Pregnancy associated AKI (PA-AKI) combined.

It was also noted that 140 patients (52.4\%) had some sort of comorbidities i.e. Diabetes Mellitus, Hypertension, ischemic heart disease and positive Hepatitis B or C.

Majority of patients required hemodialysis during management $n=190(71.2 \%)$ Table 3. Nevertheless, the overall outcome of patients was encouraging when evaluated in terms of complete recovery, partial recovery to continue as CKD or renal death requiring dialysis dependency and mortality, and is presented in Table 4, Figure 2. Overall $n=181(67.7 \%)$ patients had complete recovery to normal or baseline renal function Figure 2. With this varied presentation and causes of AKI the overall mortality was only 5.2\%.

Table 3: Need for Hemodialysis during the episode of Acute Kidney Injury among 267 patients admitted to Balochistan Institute of Nephrology-Urology Quetta (BINUQ).

\begin{tabular}{|l|l|l|}
\hline Hemodialysis & Frequency & $\%$ \\
\hline Yes & 190 & 71.2 \\
\hline No & 77 & 28.8 \\
\hline Total & 267 & 100.0 \\
\hline
\end{tabular}


Table 4: Outcome status of 267 patients with Acute Kidney Injury at the time of discharge.

\begin{tabular}{|l|l|}
\hline Outcome at the time of Discharge & Frequency (\%) \\
\hline Complete Recovery & $181(67.7)$ \\
\hline Proceeded to CKD* & $70(26.2)$ \\
\hline Expired & $11(4.2)$ \\
\hline LAMA & $5(1.9)$ \\
\hline Total & $267(100.0)$ \\
\hline${ }^{*} \mathrm{CKD}=$ Chronic Kidney Disease &
\end{tabular}

${ }^{*} \mathrm{CKD}=$ Chronic Kidney Disease

${ }^{\circ}$ LAMA $=$ Left against medical advice

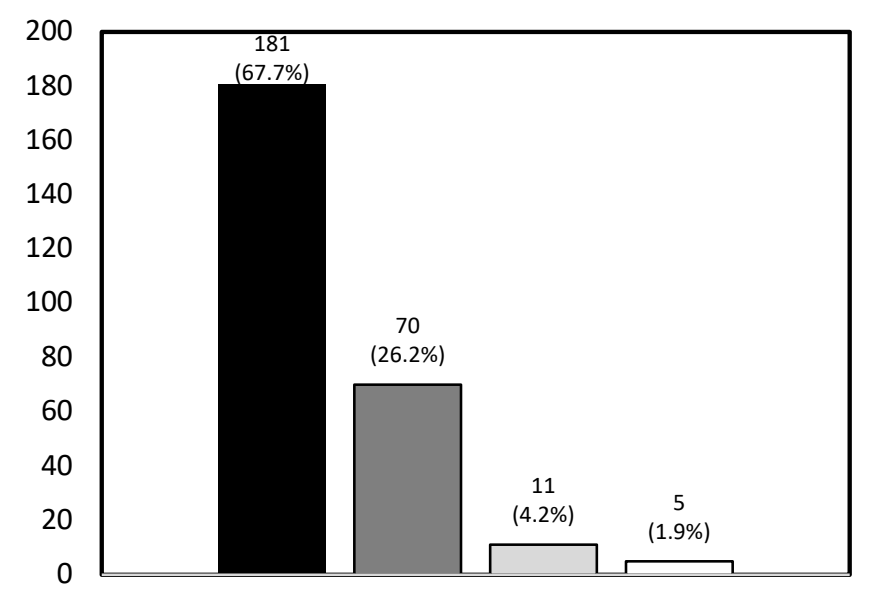

- Complete Recovery $\square$ Partial Recovery $\square$ Expired $\square$ LAMA

Figure 2: Bar graph showing the detail of outcome of 267 patients admitted with AKI. LAMA: Left against medical advice

\section{Discussion:}

We found obstetrical causes as the leading cause of AKI at our referral center and overall significant number of patients had prerenal or sepsis as the usual causes expected in a developing country. Our study also reveals a better outcome as a significant number of patients almost two third (67.7\%) recovered from AKI. In an earlier study by Naqvi SJ reported diarrheal illness and obstetrical causes as the leading cause of kidney injury. ${ }^{6}$ It is not surprising nevertheless disheartening, considering the population explosion, that after almost 40 years of the earlier publication, we are still facing the same causative factors for AKI.

In a recent study from Multan, at a medical unit (excluding the obstetrics cases) drug induced and sepsis due to various causes were the leading cause of AKI. ${ }^{9}$ Among our patients, sepsis was the cause of AKI among 7.9\% only. In another recent study from Faisalabad Bilal et al. looked at the causes of AKI among 180 pregnant females diagnosed with P-AKI at presentation to the hospital and found majority 56.6\% had puerperal sepsis. ${ }^{10}$ Among our patients with P-AKI we did not categorize further for puerperal sepsis but we suspect a significant number had sepsis as cause of P-AKI and APH or PPH was the leading cause of P-AKI among our patients. In yet another study, Bokhari et al. did a prospective follow up study of three months among 41 P-AKI patients. Majority of their patients although multiparous yet had no antenatal follow up, and sepsis was the leading cause of AKI in these patients. ${ }^{11}$ Their study highlights the health-related attitudes of pregnant females and possible areas to strengthen for ultimately improving the morbidity and mortality related to P-AKI.

A recent study by Chaudri $\mathrm{N}$ et al. from northern part of Pakistan, revealed majority of younger patients with AKI and $86.7 \%$ presenting in the late stage hence requiring dialysis. ${ }^{12}$ In this study again sepsis was the leading cause followed by drug induced AKI and others.

Our study represents the true picture of health care status related to kidney diseases especially P-AKI, that was highlighted for our geographical area as well as in other parts of Pakistan. Larger community-based studies across the country are needed to clearly identify the causes and solutions to AKI in general and particularly address the high maternal mortality and other causes of P-AKI. ${ }^{13,14}$ 
A review of the literature from the neighboring countries reveals the etiology of CAAKI close to our local literature.

Mahbub T et al. from Bangladesh reported sepsis as the leading cause of HA-AKI although it was not clear in their study that how many were actually CA-AKI. ${ }^{15}$ Similarly, recent study from India regarding CA-AKI revealed sepsis as the major cause. ${ }^{16,17}$ The study by Kaavia $\mathrm{R}$ et al. excluded the obstetrical causes whereas study by Vikrant $\mathrm{S}$ et al. had PAAKI in a small number of patients.

We would like to highlight here that majority of the causes of AKI in our population were preventable and manageable at early stage, similar to Vikrant et al. where $>60 \%$ cases of AKI were preventable. Our concern is that majority (71\%) of our patients required to undergo hemodialysis. It is well known that every episode of AKI is a potential source of adding patients to the CKD pool. ${ }^{4}$ Furthermore, it is of major concern that our patient population was mainly younger $(>50 \%)$ and this calls for a long-term study to observe the impact of AKI contributing to our CKD or dialysis population. This younger productive age group is a loss to the work force and economic burden on community health resources if they continue to be on dialysis or develop CKD. Similar trends are apparent in the developing countries. ${ }^{16,17}$

This percentage of patients requiring hemodialysis for acute renal failure was quite high among our patients as compared to studies done in developing and developed countries where this number is significantly on the lower side. ${ }^{17,18}$ Nevertheless, it is reassuring that complete renal recovery was achieved in $>60 \%$ of our patients. This whereas highlights the presence of higher number of reversible causes of AKI, but at the same time reflects the implementation of treatment protocols and international guidelines at our hospital BINUQ translating into a lower mortality among our patients, $4.2 \%$. this lower mortality is reassuring compared to other studies both local and in south Asia showing mortality trends ranging $8.7 \%-39 \% .^{11,12,16,17}$ The reason for lower mortality in our patients may be the higher number of patients with reversible causes of AKI, since majority of our patients completely recovered from AKI (67.7\%), Figure 2. A more detailed prospective trial is needed to elaborate further the etiologies of CA-AKI and devise treatment protocols as well as continuous medical education of the physicians treating such patients in community. It is only by these means that we can decrease the burden of AKI and the increasing pool of CKD and dialysis patients.

\section{Conclusion}

Our study reports a high frequency of PA-AKI and AKI due to reversible preventable causes such as obstruction and prerenal causes. Large community-based studies are needed to identify the factors and develop recommendations. In the meantime, it is the duty of nephrologists to educate the primary health care providers and obstetricians for early detection and timely referral of patients with AKI.

\section{Conflict of interest: None Declared}

\section{References}

1. Susantitaphong P, Cruz DN, Cerda J, Abulfaraj M, Alqahtani F, Koulouridis I, Jaber BL, Acute Kidney Injury Advisory Group of the American Society of Nephrology. World incidence of AKI: a meta-analysis. Clin J Am Soc Nephrol. 2013;8(9):1482.

2. Hoste EA, Bagshaw SM, Bellomo R, Cely CM, Colman R, Cruz DN, et al. Epidemiology of acute kidney injury in critically ill patients: the multinational AKI-EPI study. Intensive Care Med. 2015 Aug;41(8):1411-23.

3. Mehta RL, Cerd J, Burdmann EA, Tonelli M, G Garcia, Jha V,et al. International Society of Nephrology' s 0by25 initiative for acute kidney injury (zero preventable deaths by 2025):a human rights case for nephrology. Lancet 2015; 385: 2616 - 43

4. Bucaloiu ID, Kirchner HL, Norfolk ER, Hartle JE 2nd, Perkins RM. Increased risk of death and de novo chronic kidney disease following reversible acute kidney injury. Kidney Int. 2012 Mar;81(5):477-85.

5. Kher V, Srisawat N, Noiri E, Gharbi MB, Shetty MS, Yang L, et al. Prevention and therapy of acute kidney injury in the developing world. Kidney Int Rep (2017) 2, $544-558$

6. Naqvi, S.A.J. Regional problems in Pakistan - most prevalent kidney diseases and related problems. Proceedings from 8th Asian Colloquim in Nephrology, 1980;283-8.

7. Cugh KS, Sakhuja V, Malhotra HS, Pereira BJG. Changing Trends in Acute Renal Failure in third world countries, Chandigarh Study. Q. J.Med., 1989;73(272):1117-1123.

8. KDIGO Clinical Practice Guideline for Acute Kidney Injury. Kidney Int Suppl. 2012;2(Suppl 1):8.

9. Khan SA, Mirbahar AS, Qadir M. Acute kidney injury in hospitalized patients, frequency of various etiologies. Professional Med J 2019; 26(11):1952- 1957

10. Javaid B, Adil N, Munawar W. Factors leading to kcute kidney injury in third trimester of pregnant females visiting Allied Hospital Faisalabad. APMC 2018;12(4):288-90.

11. Bokhari SRA, Inayat F, Jabeen m, Sardar Z, Saeed S, Malik AM. Characteristics and outcome of obstetric Acute Kidney Injury in Pakistan: A Single center prospective observational Study. Cureus 10(9): e3362.DOI 10.7759/cureus.3362.

12. Chaudhri N, Shehzad MN, Qureshi MA, Masud M. Spectrum of AKI in a Nephrology Unit. J Rawal Med Col;2019;23(1): 20-24.

13. Sulaeman Z Journey of a thousand miles: harnessing mobile communications technology to solve problems in maternal health and child mortality in Balochistan, Pakistan. IEEE Pulse. 2015; Jan/Feb:28-31.

14. Naqvi R. Motherhood; blessing or a curse! A nephrologists' view. J Pak Med Assoc 2016;66(3):238-9

15. Mahbub T, Niger CR, Khanam RA, Faruq MO. Etiology and Short term Outcome of Acute Kidney Injury (AKI) in Hospitalized Patients: A Single Center Study. Bangla Crit Care J September 2019; 7 (2): 77-80.

16. Kaaviya R, Vadivelan M, Balamurugan N, Parameswaran S, Thabah MM. Community acquired AKI: A prospective observational study from a tertiary level hospital in southern India. Ind J Nephrol 2019;29(4):254-60.

17. Vikrant S, Gupta d, Singh M. Epidemiology and outcome of acute kidney injury from a tertiary care hospital in India. Saudi J Kidney Dis Transpl 2018;29(4):956-966.

18. Malhotra, K.K. Renal problems in India: most prevalent kidney diseases and related problems. Proceedings from 8th Asian Colloquim in Nephrology, 1980;289-302. 